\title{
The Mode-Coupling Theory of supercooled liquids: \\ Does it wear any clothes?
}

Glass transition of hard spheres in high dimensions, Authors: Bernhard Schmid, Rolf Schilling arXiv:1003.4559.

Mode-Coupling Theory as a Mean-Field Description of the Glass Transition Authors: Atsushi Ikeda, Kunimasa Miyazaki

arXiv: 1003.5472

A critical test of the mode-coupling theory of the glass transition Authors: Ludovic Berthier, Gilles Tarjus

arXiv:1005.0914

\section{Recommended with a commentary by Jean-Philippe Bouchaud, CFM, Paris}

The Mode-Coupling Theory (MCT) is considered by many to be the only available "first principles" approach to account for the complex dynamics of super-cooled liquids [1]. It is based on a somewhat uncontrolled closure scheme of the exact equations of motion that describe the time evolution of the dynamical structure function $S(\mathbf{k}, t)$ (i.e. the k-dependent density correlation function). The resulting self-consistent equations have several remarkable properties. They predict that as the temperature is reduced (or the density is increased) a plateau appears in $S(\mathbf{k}, t)$ at intermediate times, before the final relaxation to zero on time scales that diverge as one approaches the so-called MCT transition point, beyond which the system should be in a dynamically arrested (glass) state. Although this transition is now recognized to be an artefact of neglecting "activated processes", the promoters of MCT insist that the theory is quantitatively valid in the weakly supercooled regime, i.e. the early stages of the slowdown of the dynamics, where these activated processes should not play a major role.

There is indeed a vast body of experimental data that can be reasonably well fitted by MCT, in a restricted regime of temperatures or densities where the relaxation time increases by a factor 100 to 1000 from its value in the lowviscosity liquid. One of the important features of the theory is that all dynamical properties can be computed from the knowledge of the static structure factor, $S(\mathbf{k}, t=0)$. This leads to falsifiable predictions, concerning for example the 
detailed $\mathbf{k}$ dependence of the height of the plateau in $S(\mathbf{k}, t)$, or the non trivial ("reentrant") phase diagram of some colloids. These predictions are indeed often found to be in quantitative agreement with experimental or numerical results.

From a theoretical point of view, however, several nagging worries have been lingering around for a long time. One of them is the absence of a well defined limit in which the MCT equations for liquids are exact. It is reassuring that mean-field equations for magnets can be derived in the limit of large spatial dimensions. Other self-consistent equations in condensed matter physics can be justified by considering a large spin limit, or a large number of component limit, etc. In the case of MCT, a field-theoretical formulation - that would naturally lend itself to consistent approximation schemes - is fraught with difficulties, and is still unresolved [2,3]. On the other hand, a mean-field treatment of a family of spin-glasses does lead to dynamical equations with a mathematical structure akin to the MCT equations for liquids, suggesting that there should indeed be a way to formulate MCT as a mean-field approximation of some sort.

Unfortunately, some recent papers suggest that the above problems might be serious. The quantitative agreement between MCT for supercooled liquids and numerical/experimental results should not be restricted to three-dimensional systems only. There is a priori no reason to believe that the approximations used to derive MCT should not work in other dimensions as well. This is what two groups have independently investigated [4,5], by studying hard sphere systems in dimension $d=4$ up to $d=\infty$. (Hard spheres in $d=3$ have been one of the testbed of MCT). Unfortunately, the results are not good; inconsistencies appear as soon as $d>4$, and the situation becomes worse and worse as $d$ increases. This suggests that the quantitative success of MCT in $d=3$ might be partly coincidental. But a more disturbing predicament stems from the very recent work of Berthier \& Tarjus [6], who took on the basic tenet of MCT: that the dynamics is entirely encoded in the static structure factor $S(\mathbf{k})$. They came up with a model of glass former where the attractive part of the interaction between particles can be switched on and off in such a way that $S(\mathbf{k})$ is left unchanged. But the dynamics is seen to change radically between the two systems (the one with attraction being much slower than the one without), even in the weakly supercooled regime where MCT should hold!

Can MCT still save its head? It may after all be quixotic to look for quantitative agreement between a mean-field, approximate theory and empirical/numerical data. While MCT is probably not the one-size fit-all theory that was first hoped, the physical scenario underlying the MCT equations does capture an important aspect of the glass transition: the crossover between an essentially free motion of the particles to a activated motion between well separated local minima of the energy landscape. With A. Andreanov and G. Biroli [7], we have argued that MCT should be interpreted as a Landau theory describing this topological change of the energy landscape. In other words, while the mathematical structure of the equations should be generic and have a broad range of validity, the value of the 
parameters involved in the equation should not be taken too seriously.

But even within this restricted interpretation, activated effects are still left out of the theory, and are clearly important to understand the dynamics at long times, even for relatively high temperatures. Furthermore, as with all Landau theories, critical fluctuations become dominant close enough to the transition below some dimension, which turns out to be $d_{c}=8$ for MCT. Whether or not all these complications leave a sliver of the parameter space where MCT provides genuinely testable predictions, is at this stage an open problem.

[1] for a review, see: W. Götze, Complex Dynamics of Glass-Forming Liquids, A Mode-Coupling Theory, Oxford University Press (2009).

[2] A. Andreanov, G. Biroli, and A. Lefèvre, J. Stat. Mech. P07008 (2006).

[3] B. Kim and K. Kawasaki, J. Stat. Mech. (2008) P02004

[4] B. Schmid, R. Schilling, Glass transition of hard spheres in high dimensions, arXiv:1003.4559, Phys. Rev. E 81, 041502 (2010).

[5] A. Ikeda, K. Miyazaki, Is Mode-Coupling Theory the Mean Field Theory of the Glass Transition?, arXiv:1003.5472

[6] L. Berthier, G. Tarjus, A critical test of the mode-coupling theory of the glass transition, arXiv:1005.0914

[7] A. Andreanov, G. Biroli, J.-P. Bouchaud, Europhys. Lett. 8816001 (2009). 